\title{
A NEW ANATOMICAL NOTATION OF THE VISUAL FIELD
}

BY

DR. M. URIBE Troncoso

NEW YORK

THE examination of the visual field deserves a more important place in the routine examination of the eyes than it now holds. Not only the beginner but the practising ophthalmologist as well, are deterred from a wider use of this method by the many complexities and loss of time its practical application entails. For this reason the simplification and improvement of our present technique will do much in promoting a better understanding and larger application of this important diagnostic agency.

One of the first technical difficulties the beginner has to deal with, is the way of recording the visual field on the chart. As mapped now the findings in the perimeter need to be inverted twice. First comes the physiological inversion by which the image formed in the retina is projected to the opposite side of the eye. This requires the readings of the position of the test carrier to be made on the side of the perimeter opposite to the part of the retina under examination. The second inversion is made necessary by the habit of considering the visual field in relation with the position of the examiner's eye, and requires a total transfer of the findings for charting the right side of the patient's eyes to the right side of the observer's eye, the left side to the left, etc. This double inversion is the result of a faulty mental habit and is in complete violation of the anatomical rule which requires the subject to be considered always standing in front of the observer.

In a previous paper ${ }^{(1)}$ I have emphasized the confusion and misunderstanding that the departure from this basal anatomical rule has produced in all ophthalmological descriptions and the advantages to be derived from the change to one standard position instead of two. The present method of recording the field of vision was probably originated by the primitive use of the blackboard and afterwards of the campimeter, in which the examiner places himself behind the patient. The limits of the field and scotomas are drawn on the blackboard in relation to the eyes of both, considered in the same position on the space, and the mapping, when necessary, is very simple, as it only requires a similar drawing on a reduced scale. This method had the advantages of enabling the observer to visualize the conditions on which the visual cone of the patient is projected into space, 
and to refer all changes to the examiner's own right and left sides. With the advent of the perimeter, however, conditions for the examination of the fields changed entirely. The observer had to stand in front of the patient for moving the test carriers, reading the findings on the arc, and watching the central fixation of the eye. This change of position immediately required a readjustment of the mental picture of the visual cone of the patient, now extending in front of the observer, the right side of the patient corresponding to the left of the physician. The physiological inversion of the retinal images could be more clearly understood and recorded on the arc of the perimeter in this position, the larger temporal field corresponded to the larger extent of the nasal retina, and the restricted nasal field to the shorter area of the temporal retina; the blind spot being about in the centre of the percipient layer of this membrane (Elliot). But when the findings in the arc were to be recorded on the chart, no change was made to meet the new conditions, and the old schemes were used, with the result that a second inversion became necessary to map the temporal field A of the patient's eye (Fig. 1), at the corresponding point $B$ of the observer's eye. This second inversion although' relatively easy for a trained ophthalmologist, is difficult and requires a considerable mental effort for the beginner. The transfer is much easier in the four cardinal points, viz. : temporal, nasal, upper and lower meridians, but for intermediate positions, $30^{\circ}$ or $60^{\circ}, 300^{\circ}$ or $330^{\circ}$, which need to be recorded at the same inclination on the corresponding side of the observer's eye, the difficulty increases a great deal, especially if, as frequently happens, the graduation of the meridians in the card is different from those in the perimeter.

In order to avoid the inevitable confusion and misunderstanding of the double inversion, the self-registering perimeters were devised. In the simpler instruments the chart is carried by a disc which rotates with the arc, and the markings are made on the side of the same colour, red or white, of the figures of the arc. Even with this perimeter, however, when the observer, for saving time, tries to use both sides of the arc alternately, the same embarrassment and confusion arises in regard to the place for marking and the inclination of the meridians. Only in the more expensive and complicated models are the records made automatically accurate.

The difficulties of this faulty, irksome method of recording the visual field are particularly evident when the student tries to determine to which part of the field a given defect or lesion in the retina corresponds. The mental habit of considering the relative positions of the papilla and the macula as we see them with the ophthalmoscope, makes it difficult for the observer to 
transpose immediately to his own eye the position of the blind spot in the patient's fields. With the ophthalmoscope the papilla is seen inward and a little upward of the macula, and

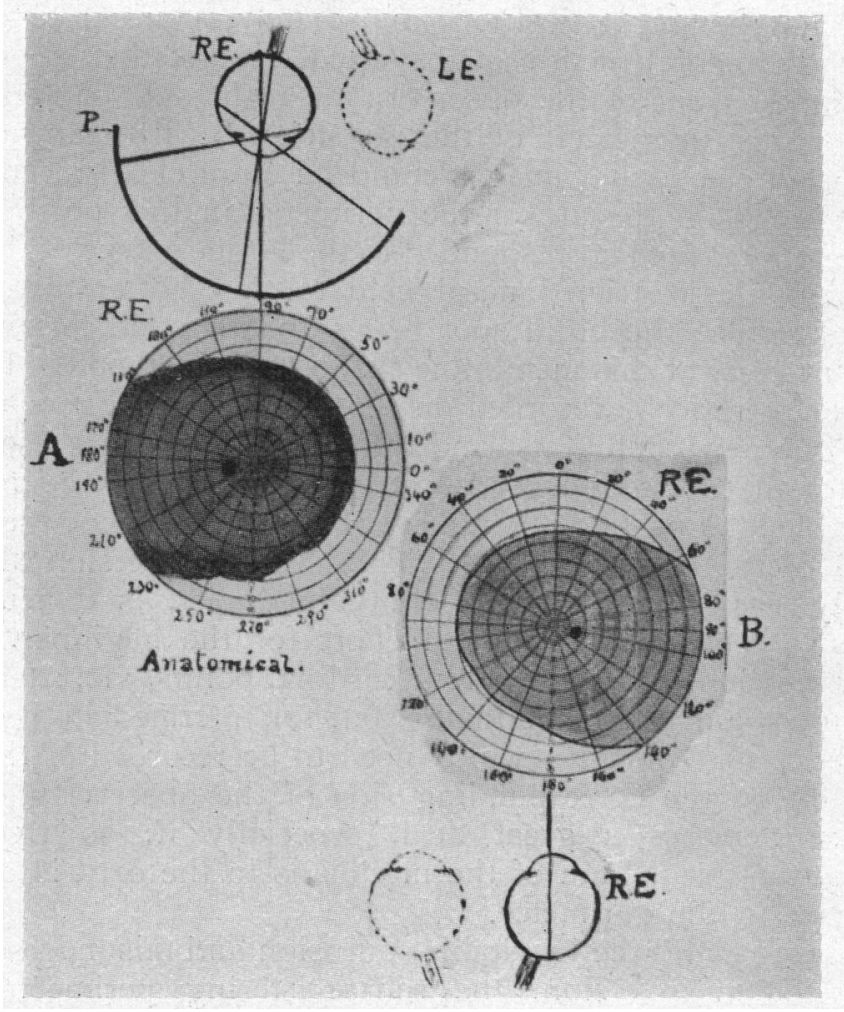

FIG. 1.

A. Position of the field of vision in front of the patient's eye in the anatomical method. R.E., Right eye. P., Perimeter. A, temporal side of the field. The graduation of the meridians is the same as in the trial frame.

B. Temporal side of the field of vision as recorded at present, facing the observer's eye. The plotting needs a second inversion and is confusing and misleading.

according to the law of visual projection it is easily located on the arc of the perimeter, outside and a little below the fixation point. But when we try to mark its position in the diagram it will be necessary to carry what in the eye ground is at the patient's right to our own right, and afterwards imagine its relations with the other parts of our own fundus. 
It is even more arduous to find the place which in the patient's field of vision will correspond with a characteristic change found with the ophthalmoscope. A thrombosis of the superior nasal artery of the right eye, for instance, will produce a sector-like defect in the opposite side of the field, that is in the inferior

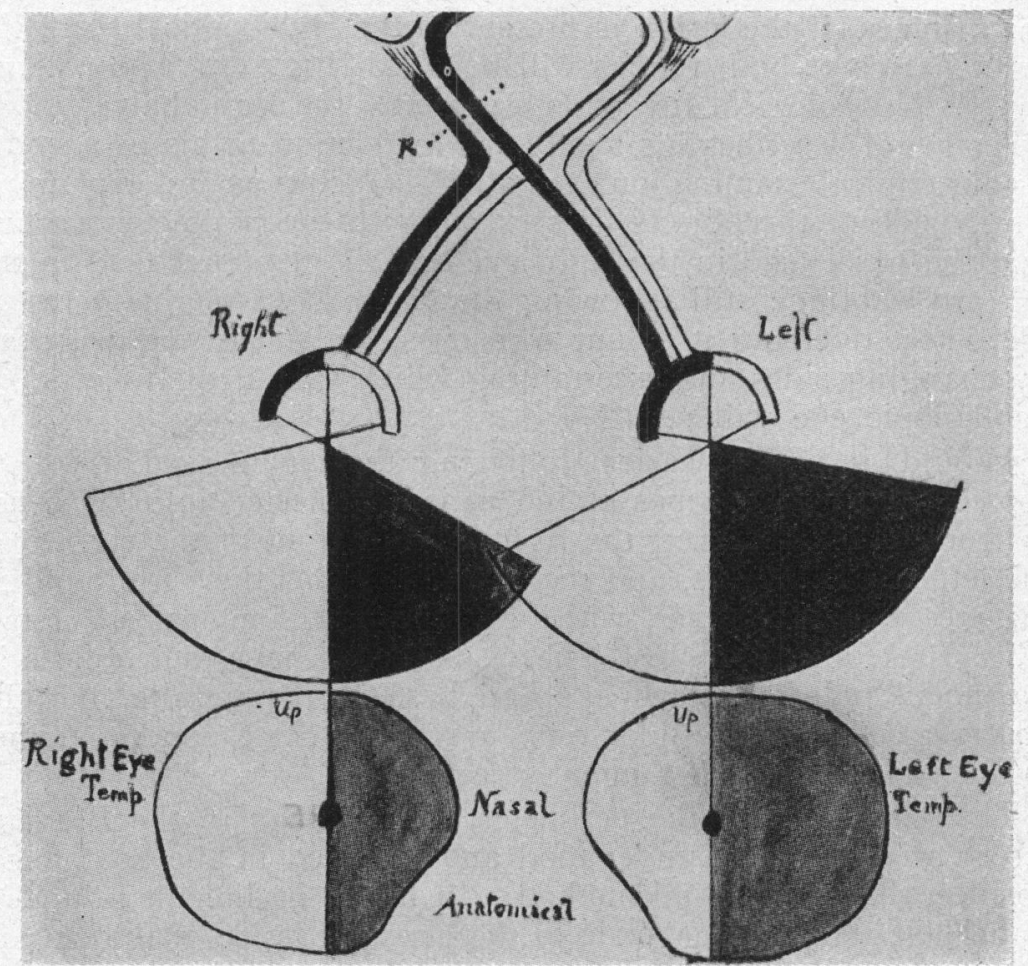

Fig. 2.

Schematic representation of the optic pathways, fields of vision on both sides as projected in the space in front of the observer, and records of the fields in the anatomical position.

temporal quadrant. With the perimeter this defect will be immediately found in its proper place, but if the observer tries to visualize the ophthalmoscopic lesion and then represent to himself the defect produced in the field, he will have to transfer the lesion first to his own eye, and then locate the defect in the opposite side according to the law of visual projection.

Probably on account of the early use of the campimeter and the facility of representing the pathological changes in the patient, the description of diseases of the central nervous system has also been 
made considering the observer as standing behind the patient. The well-known diagrams of the central nervous system, the tracts, optic nerves and correlation of the two retinas are always represented in the unanatomical position; the oniy advantage of this is to be able to visualize the lesions as if they existed in the physician's own organs and designate the right and left sides by the same names. This has, however, numerous disadvantages. In hemianopsia, for instance, if the lesion is present in the right optical tract, the right halves of both retinas will be blind (Fig. 2). The patient, by the physiological inversion, sees only the right halves of all objects and describes his symptoms in relation to his own body. In our clinical examination the patient is facing us and explaining which side of the objects he sees. With the perimeter we find that the nasal field in the right eye and the temporal field in the left are wanting; still following the custom, we ought to record the defect referring it to our own eyes, and make the diagnosis by changing sides and ascertaining which side of the objects our hemianopic eye will see.

Would it not be easier, following the anatomical rule, to consider the visual cones of the patient projected into the space in front of the observer, the right field in front of the right eye, the left before the left, and record the fields as they are found in the arc of the perimeter, with only one inversion instead of two (Fig. 1, A, and Fig. 2)? The visual cones have their apices in the nodal points of each eye and base in the infinite, but the observer needs to consider only a section, corresponding either to the perimeter or the tangent screen.

The same as with the perimeter, the findings in the Bjerrum screen will be better understood and recorded in the anatomical position than by the old method. In fact it is difficult to understand how the plotting made in the graduated side of the curtain facing the observer, was not immediately recorded in a diagram exactly as it was found, instead of making the second inversion to the examiner's eye.

No change in our instruments is necessary for the new method, except that the campimeter should be made of cloth for thrusting pins and marking the limits and defects in the field on the side facing the operator.

With the adoption of the new anatomical field it would be important to standardize the notation of the meridians in the charts, which at the present time is very confusing. In some charts $0^{\circ}$ is placed aboye, ${ }^{\prime} 180^{\circ}$ below, and $90^{\circ}$ on either side of the horizontal meridian for both eyes. In others for the right eye the $0^{\circ}$ is above, $90^{\circ}$ at the left of the patient, $270^{\circ}$ to his right and $180^{\circ}$ below. For the left eye $90^{\circ}$ is at the right of the patient and 
$270^{\circ}$ to the left, the vertical meridian being the same as in the - other eye.

In America the most accepted notation is $90^{\circ}$ above, $270^{\circ}$ below, and the $0^{\circ}$ at the left side of the patient. In the anatomical field it will be desirable to adopt the last-mentioned notation, and represent the inclination of the meridians exactly in the same way as the axis of the cylinders in the trial frame, that is the $0^{\circ}$ always at the left of each eye of the patient, $180^{\circ}$ to the right, $90^{\circ}$ above, and $270^{\circ}$ below (Fig. 1, A). In this way it will be easy

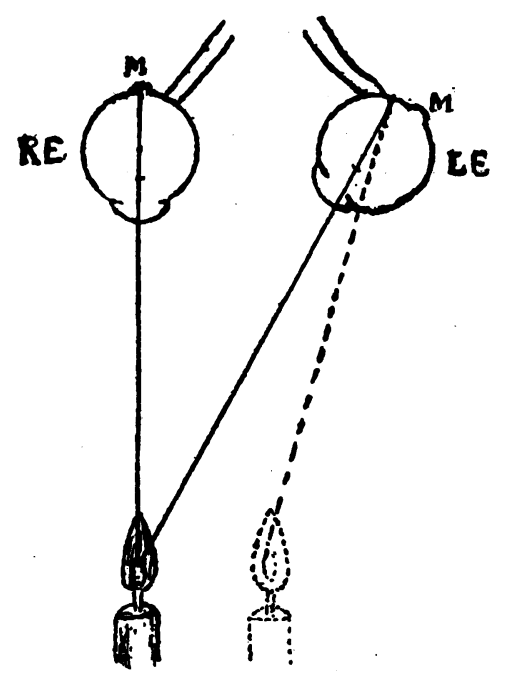

FIG. 3.

Homonymous diplopia. The deviation of the left eye inward makes the image of the candle fall at the inner side of the macula, M, and this image is projected outward, where a false image is seen at the left.

to find the inclination of every meridian and draw an accurate diagram even on a provisional chart, by comparison with the trial frame.

I am well aware that this complete change in the mapping of the visual field will find a great opposition amongst ophthalmologists, who being trained in the double standard position, have acquired the habit of the mental process required to make the inversion quickly and without any mental strain. They will find it unnecessary or even inadvisable to depart from their habits, but for the beginner in ophthalmology to be taught in the anatomical position to which he has been accustomed, will mean much time saved and much effort spared.

The transition between the old and new method is very simple. New charts may be printed with the same diagram they have at 
present, not exactly inverted, but as seen by translucency; the temporal side of the chart in front of the temporal side of both orbits of the patient. Even the old charts can be put to service, simply changing sides, the right eye being used for the left and vice versa; but of course the graduation in the meridians will become inverted, which is not desirable.

Fixation Field.-The monocular and binocular fields of fixation will be more easily understood and recorded in the anatomical position, than after the inversion necessary for transferring them to the position of the observer's eye.

In paralytic strabismus the examination of the binocular field of fixation with the tangent screen in the six cardinal directions will detect the limitation of movement, the extent of the field for single and double vision, the kind of diplopia, and the separation of the two images, which can be plotted on the white side of the screen and recorded on the anatomical card without any inversion. This will give the student a much better idea of the situation of the false image in space and its relations with the retina of the patient, as he can visualize by the deviation of the optical axis the new location of the image outside the macula and the direction in which the false image will be projected (Fig. 3).

It is a well-known fact that the chapter on abnormal motility of the eye is considered one of the most difficult to grasp by the student. The normal anatomy and physiological action of the extraocular muscles are taught with the cadaver or subject in the standard anatomical position, but the abnormalities in the function are considered not in the same way, the proper scientific method, but by reversing the findings to the observer's own eye, thus creating a double system for the study and interpretation of these complicated symptoms with inevitable confusion and misunderstanding.

In a paper presented to the Ophthalmological Section of the American Medical Association in May of this year, Dr. Luther C. Peter, of Philadelphia ${ }^{(2)}$, endorses this anatomical method, and gives added reasons for the adoption of one universal standard instead of two. The same author in a personal communication, states also his belief in the necessity of changing the recording of the field of vision to the more scientific and accurate anatomical position.

\section{REFERENCES}

1. M. Uribe Troncoso. - Anatomical versus Ophthalmological Nomenclature. Trans. Internat. Con. Ophthal., Washington, 1922.

2. Luther C. Peter.-Anatomic Method of studying and recording the Motility of the Eyes. Trans. Sec. Ophthal., Amer. Med. Assoc., May. 1925. 